\title{
Super-Resolution Optical Microscopy in Biology
}

\section{Markus Sauer $^{1 *}$}

1. Department of Biotechnology and Biophysics, Biocenter, University of Wuerzburg, Am Hubland, 97074 Wuerzburg, Germany

*Corresponding author, m.sauer@uni-wuerzburg.de

Imaging technologies are central platforms that drive fundamental research in virtually all disciplines across the biological and medical sciences. By seeing how a system looks, function can be understood and comparison made between healthy and unhealthy systems whether they are plant, animal or human and whether they are single cells, tissues, whole organisms or populations. The unique combination of molecule-specific labelling, minimal perturbation, and three-dimensional (3D) imaging makes fluorescence microscopy presently the most popular imaging modality in cell biology. The level of detail images contain is determined by the achievable image resolution and should match the characteristic feature size of the object studied. In cell biology, we encounter objects ranging from a size of microns to nanometres, and we are interested to understand how they are organized and how they interact with their environment. In order to obtain a comprehensive mechanistic picture of cellular processes, we need information at the level of cellular components - organelles, protein complexes and single proteins which are of a size from a few to many nanometers. Understanding how biomolecules are organized into complexes and the dynamics of this organization are essential in order to understand how a cell functions, and which mechanisms occur in the case of a dysfunction or disease. In this context, a spatial resolution in the range of the size of biomolecules is needed [1].

Unfortunately, diffraction posed a historic limit on the resolution power of fluorescence microscopy and limited the minimal distance at which two objects can be discerned to about half of the wavelength of light [2]. This is largely sufficient to study large-scale features of cells and tissue, and much of our todays understanding of how cells are organized and biomolecules interact has been gained by standard light microscopy techniques. The rise of super-resolution microscopy, i.e. fluorescence microscopy techniques that achieve a substantially better spatial resolution down to a few nanometers, has opened another window to study cellular processes: now, it is possible to investigate how biomolecules are organized and how they interact at the nanometer scale, and from that to understand how a misconfiguration of the molecular architecture and intermolecular interactions can lead to various forms of disease. For sure, super-resolution microscopy techniques are just about to enter topics of health and disease, and many more work in different directions is needed. However, we can anticipate that from a "nanoscopic" inspection of how proteins organize and interact inside a living cell, we will be adding significant information for an improved understanding and treatment of diseases.

Among all powerful high-resolution imaging techniques introduced [3-5], single-molecule localization microscopy (SMLM) methods such as (F)PALM [6,7] and (d)STORM [8-10] excel because they deliver single-molecule information about molecular distributions, even giving absolute numbers of proteins present in subcellular compartments. This provides insight into biological systems at a molecular level that can yield direct experimental feedback for modelling the complexity of biological interactions. Since its invention, the technique of localizing individual fluorescent molecules in a densely labeled sample by exploiting on-off transitions in fluorophores has been extended to live cell, multicolor and 3D imaging $[11,12]$. 
I will show how single-molecule localization microscopy methods such as $d$ STORM can be used advantageously to visualize the distribution and quantify the absolute number of tumor-associated antigens on healthy and tumor cells. A paradigm shift is currently taking place in cancer medicine (hematology \& oncology). It has been realized and clinical-proof-of concept has been obtained that immunologic anti-cancer treatments with antibodies and tumor-specific $\mathrm{T}$ cells are highly effective and indeed capable of curing advanced, end-stage malignancies that are resistant to conventional treatments. However, a critical challenge that arises in both research and clinical practice is that the sensitivity of antibodies and $\mathrm{T}$ cells is much higher than the sensitivity of detection methods such as immunohistochmistry (IHC) and flow cytometry (FACS) that are available to actually screen the patient's tumor for expression of the respective antigen. Here, $d$ STORM can be used to quantitatively map tumorassociated membrane receptors, ideally the entire tumor receptome, using commercially available fluorescently labeled antibodies (Fig. 1).
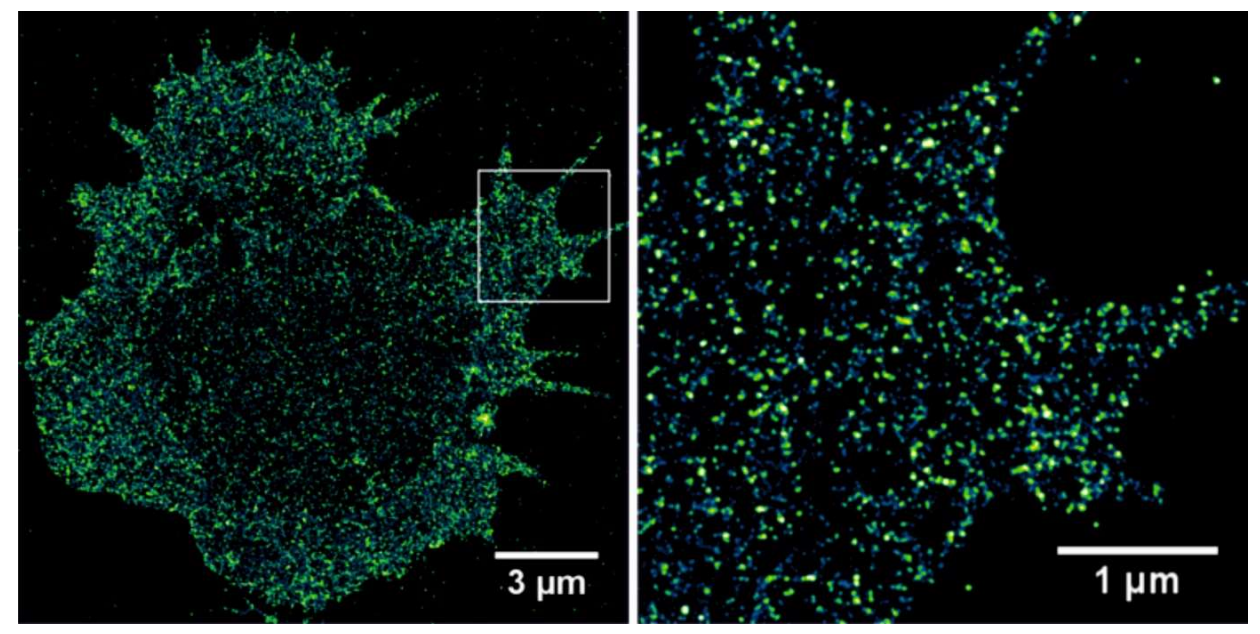

Figure 1. $d$ STORM

visualizes the distribution of CD19 receptors on the plasma membrane of primary tumor cells.

This provides important information about possible tumor-specific surface targets for antibodies and $\mathrm{T}$ cells. That is, $d$ STORM imaging of the receptome of primary tumor cells enable the detection and selection of single or combinations of tumor antigens, and based on molecule density per cell, the selection of the optimal treatment modality for an individual patient.

Another biomedical field which can profit from the distinct single-molecule sensitivity of $d$ STORM is neurosciences. One of the major challenges in science is to understand the human brain and despite ongoing research it might be argued that our insights so far remain rather modest. Beginning with the identification of neurons as elementary building blocks of the nervous system, continuous technological advances in experimental resolving power have provided us with insights into signal transduction between neurons, which occurs at specialized neuronal sub-compartments, termed synapses. Synapse formation is a highly regulated process that requires the coordination of many cell biological events. Decades of research have identified a long list of molecular components involved in assembling a functioning synapse. Yet how the various steps, from transporting synaptic components to adhering synaptic partners and assembling the synaptic structure, are regulated and precisely executed during development and maintenance is still unclear. 
There is overwhelming evidence that defects in the synaptic function and in the fine structure of synapses are involved in the generation of severe neurological and psychiatric diseases. Here, again technical limitations hindered to date a better understanding of the structure-function relationships in the healthy and diseased brain. In a recent collaborative effort, we introduced a novel procedure to determine endogenous protein numbers in tissue by $d$ STORM to analyze, e.g., the molecular organization of the synaptic protein Bruchpilot and to estimate its copy number per presynaptic active zone in situ at the Drosophila neuromuscular junction (NMJ) [13]. We found that the active zone cytomatrix (CAZ) is composed of units containing $\sim 137$ Bruchpilot (Brp) proteins, three quarters of which are organized into about 15 heptameric clusters.

Furthermore, we applied 3D- $d$ STORM to map protein distributions with about $20 \times 20 \times 60 \mathrm{~nm}$ spatial resolution in mouse brain cryosections. In up to $25 \mu \mathrm{m}$ thick cryosections we recorded en bloc thousands of neuronal subcompartments abberation free in volumes up to $28 \times 30 \times 9.5 \mu \mathrm{m}^{3}$. Using highly specific anti-Bassoon antibodies we measured protein clusters with distinct size, number and density in mouse hippocampus. Applying sequential imaging in brain sections of Thy1-mEGFP (Ls1) mice we found largest Bassoon clusters to be located in in CA3-MF boutons. To conclude, single-molecule localization microscopy by $d$ STORM might pave the way for a refined understanding of the molecular organization of synapses and ultimately unravel how nature encodes function at the molecular level.

\section{References:}

[1] M Sauer and M Heilemann, Chem. Rev. 117 (2017) 7478.

[2] E Abbe, Arch. Mikrosk. Anat. 9 (1873) 413.

[3] SW Hell and J Wichmann, Opt. Lett. 19 (1994) 780.

[4] SW Hell, Science 316 (2007) 1153.

[5] MG Gustafsson, J. Microscopy 198 (2000) 82.

[6] E Betzig et al, Science 313 (2006) 1642.

[7] ST Hess, TP Girirajan and MD Mason, Biophys. J. 91 (2006) 4258.

[8] MJ Rust, B Bates and X Zhuang, Nat. Methods 3 (2006) 793.

[9] M Heilemann, et al, Angew. Chem. Int. Ed. 47 (2008) 6172.

[10] M Heilemann, S van de Linde, A Mukherjee, M Sauer, Angew. Chem. Int. Ed. 48 (2009) 6903.

[11] S van de Linde, et al, Nat. Protocols 6 (2011) 991.

[12] TJ Gould, VV Verkhusha and ST Hess, Nat. Protocols 4 (2009) 291.

[13] N Ehmann, et al, Nat. Communications 5 (2014) 4650. 
https://doi.org/10.1017/S1431927618013995 Published online by Cambridge University Press 